\title{
Note su Boldoriella del gruppo carminatii: Boldoriella silvanae stat. nov. e osservazioni sistematiche sulle specie affini (Coleoptera Carabidae Trechinae)
}

\begin{abstract}
Riassunto: Nel presente contributo è modificato il rango tassonomico di Boldoriella carminatii silvanae Monzini, 1995, elevato a Boldoriella silvanae, stat. nov. Vengono anche riesaminate numerose popolazioni di Boldoriella carminatii (sensu lato), insieme con altre due specie strettamente affini: Boldoriella manzoniana Monzini, 1995 e Boldoriella concii Monguzzi, 1982 (sensu lato). Le quattro specie citate sembrano rappresentare una linea monofiletica per le affinità morfologiche e le rispettive vicinanze distributive. Per tale ragione, vengono qui aggregate in un "gruppo carminatii".
\end{abstract}

\begin{abstract}
Notes on Boldoriella of the carminatii species group: Boldoriella silvanae stat. nov. and taxonomy of related species. (Coleoptera Carabidae Trechinae).

In this contribution, the taxonomic status of Boldoriella carminatii silvanae Monzini, 1995, is changed to Boldoriella silvanae, stat. nov. Many populations of Boldoriella carminatii (sensu lato) are reviewed to, as well as two closely related species: Boldoriella manzoniana Monzini, 1995, and Boldoriella concii Monguzzi, 1982 (sensu lato). These four species, because of their morphological affinities and distribution nearness, seem to form a single monophyletic lineage. For that reason, they are here aggregated into a carminatii species group of the genus Boldoriella.
\end{abstract}

Key words: Coleoptera, Carabidae, Boldoriella, silvanae, carminatii, taxonomy, distribution.

\section{INTRODUZIONE}

Boldoriella carminatii fu descritta quasi un secolo fa, nel 1917, da Agostino Dodero come Trechus (Duvalius) carminatii. Successivamente sono state scoperte, descritte e variamente interpretate altre specie e sottospecie riconducibili alla stessa linea filetica in base alla somiglianza di caratteri morfologici esterni e a livello dell'edeago (cfr. Monguzzi, 1982). Alcune di queste si collocano in posizione periferica rispetto alla distribuzione di $B$. carminatii (Boldoriella silvanae stat. nov., Boldoriella manzoniana) (Monzini, 1995), altre risultano simpatriche e sintopiche con B. carminatii. Da citare Boldoriella concii (Monguzzi, 1982), che per lungo tempo fu addirittura considerata come una semplice popolazione di Boldoriella carminatii (cfr. Boldori, 1931; Ghidini, 1932), e solo in seguito fu riconosciuta come specie distinta, anche in considerazione dell'accertata simpatria e sintopia tra le sue sottospecie: B. concii folinii (Monguzzi, 1982) con $B$. carminatii bucciarellii (Monguzzi, 1982), e B. concii culminicola (Monguzzi, 1982), con la forma tipica di B. carminatii. Nel presente contributo, è presentata una revisione dei caratteri delle specie citate, per meglio chiarire la loro posizione e identificazione.

\section{Materiali e Metodi}

Sono stati esaminati in totale 278 esemplari, provenienti dalle seguenti località.

- Boldoriella carminatii: Grotta Tomba del Polacco 1003 Lo, Grotta Lacca del Palio 3722 Lo, M.te I Canti Fuipiano Imagna (MSS), Grotta Bus del Boter 1005 Lo, Grotta -1 condotta forzata (innominata) presso Corna Imagna, Grotta -2 (innominata) presso Corna Imagna, Grotta dei Morti 1042 Lo, Grotta di Val d'Adda 1044 Lo, Culmine San Pietro LC (MSS), Miniera la Passata del M.te Resegone, Miniera del M.te Misma Abbazia BG;

- Boldoriella carminatii bucciarellii: Dossena BG (MSS), Grotta Lacca di Sponcc 3515 Lo, Miniere della Plassa M.te Arera BG, Miniera di GornoOneta BG, Miniera -1 e Miniera -2 di Parre BG;

- Boldoriella concii: Grotta di Vallasnina 1001 Lo;

- Boldoriella concii folinii: Val Parina BG (MSS), Dossena BG (MSS);

- Boldoriella concii culminicola: Culmine San Pietro LC (MSS), M.te i Canti presso Fuipiano BG (MSS);

- Boldoriella silvanae: Colle Brianza presso Camsirago LC (MSS), M.te Crocione presso Dozio LC (MSS);

- Boldoriella manzoniana: miniere del M.te Coltignone, Pian dei Resinelli LC. 
L'esame microscopico è stato effettuato con microscopio ottico Optika, modello Mic12.

Le macrofoto degli habitus e degli edeagi sono state effettuate mediante fotocamera digitale Canon montata su microscopio ottico Optika.

\section{RISULTATI}

Le quattro specie oggetto del presente contributo mostrano chiare affinità reciproche nella morfologia dei rispettivi edeagi, caratterizzati dalla forma gracile e allungata del lobo mediano, con curvatura più o meno accentuata a seconda delle specie, ma sempre evidente (Figg. 1-4), oltre che per la morfologia esterna (Figg. 5-8), caratterizzata in modo particolare dalla forma delle elitre ovoidali, e del pronoto che presenta margini laterali sinuati, con angoli basali acuti e divergenti. In base a tali elementi, oltre che per la contiguità o la sovrapposizione dei rispettivi areali, questo complesso di specie chiaramente monofiletico viene raggruppato e definito come "gruppo di Boldoriella carminatii". Inoltre, in base a queste considerazioni, l'interpretazione originaria di Boldoriella silvanae quale sottospecie di $B$. carminatii, pur con molti dubbi espressi all'atto della sua descrizione, viene riconsiderata e aggiornata quale specie valida e distinta, in quanto il grado di diversità, sia nella forma dell'edeago e della lamella copulatrice, sia nei caratteri esterni, equivale a quello che intercorre tra tutte le altre specie del gruppo.

Il rango del taxon è quindi aggiornato in: Boldoriella carminatii silvanae $=$ Boldoriella silvanae stat. nov.

\section{TABELLA DI DETERMINAZIONE DELLE SPECIE DI}

\section{BOLDORIELLA DEL GRUPPO CARMINATII}

1 Elitre con omeri molto arrotondati o sfuggenti... 2

- Elitre con omeri distintamente angolosi .......... 3

2 Omeri arrotondati, strie elitrali superficiali, tegumenti lucidissimi, dimensioni maggiori (4.8-6.5 $\mathrm{mm}$ ), tempie convesse, pronoto trasverso con lati fortemente arcuati, edeago allungato e ricurvo con "bottone" apicale rilevato, lamella copulatrice poco allargata. Dalla Val Sassina alla Val Brembana e alla bassa Val Seriana (f. typ.); più a Nord, nel complesso dei Monti Arera, Alben, Secco (ssp. bucciarellii Monguzzi)...carminatii (Dodero) (sensu lato) - Omeri sfuggenti, strie elitrali profonde e punteg- giate, tegumenti moderatamente opachi, dimensioni medie (4.5-5.8 mm), tempie spianate, solchi frontali incompleti, pronoto non trasverso con lati poco arcuati, edeago allungato e diritto con uncino apicale pronunciato e "bottone" apicale poco rilevato, lamella copulatrice allargata. Diffusa fra i Monti Grignetta e Coltignone.

manzoniana Monzini

3 Antenne distese all'indietro non superanti la metà delle elitre, tegumenti lucidi, dimensioni minori (3.8-5.2 mm), edeago gracile, allungato e ricurvo, con "bottone" apicale rilevato, lamella copulatrice allungata e poco allargata. Diffusa nella bassa Val Seriana (f. typ.), nell'alta Val Seriana sui M.ti Alben-Arera (ssp. folinii Monguzzi), e a ovest della Val Brembana tra il Culmine San Pietro e la Valle Imagna (ssp. culminicola Monguzzi)

concii Monguzzi (sensu lato)

- Antenne distese all'indietro superanti ampiamente la metà delle elitre, tegumenti opachi e iridescenti per la microscultura forte, a maglie reticolari, dimensioni medie (4.6 - 5.6), edeago moderatamente allungato, poco ricurvo, con "bottone" apicale poco rilevato, lamella copulatrice breve, larga, con apice aguzzo e saliente. Diffusa sui monti dell'alta Brianza, tra i fiumi Adda e Lambro silvanae Monzini (Tab. 1).

\section{COROLOGIA E CONSIDERAZIONI ZOOGEOGRAFICHE}

Boldoriella carminatii presenta una distribuzione molto ampia (Fig. 9), che si estende in senso Est-Ovest dal solco del Lago d'Endine - Val Cavallina fino alla Valle dell'Adda, mentre in senso Nord-Sud è estesa dal lembo più settentrionale delle Prealpi calcareo/dolomitiche (che coincide con i maggiori rilievi orobici: Zuccone Campelli (Casale, 1973), Pizzo Arera e Monte Secco), fino ai rilievi pedemontani. Di questa specie sono state censite numerose popolazioni, presenti in varie grotte naturali e cavità artificiali; a quote più elevate è reperibile occasionalmente anche in Ambiente Sotterraneo Superficiale (MSS). La specie presenta una notevole costanza di caratteri nelle popolazioni occidentali che sono piuttosto uniformi, come anche quella estrema orientale e marginale del Monte Misma, oltre il Fiume Serio.

Presenta invece una certa variabilità nella media Val Seriana, fino a raggiungere la massima 


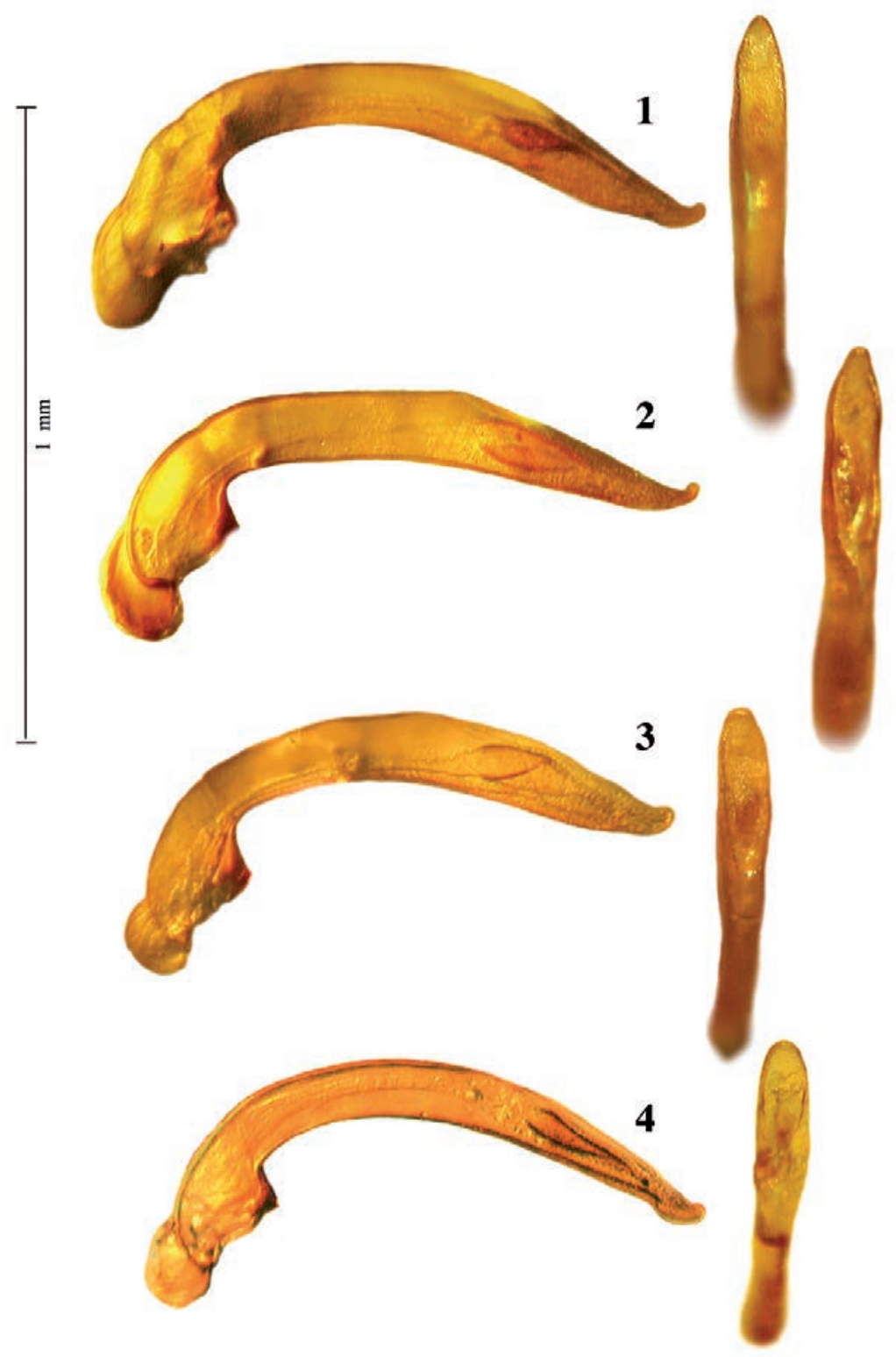

Figg. 1-4. Edeagi in visione laterale e dorsale di esemplari ổ topotipici di: 1 - Boldoriella carminatii; 2 - Boldoriella manzoniana; 3 - Boldoriella silvanae; 4 - Boldoriella concii. 

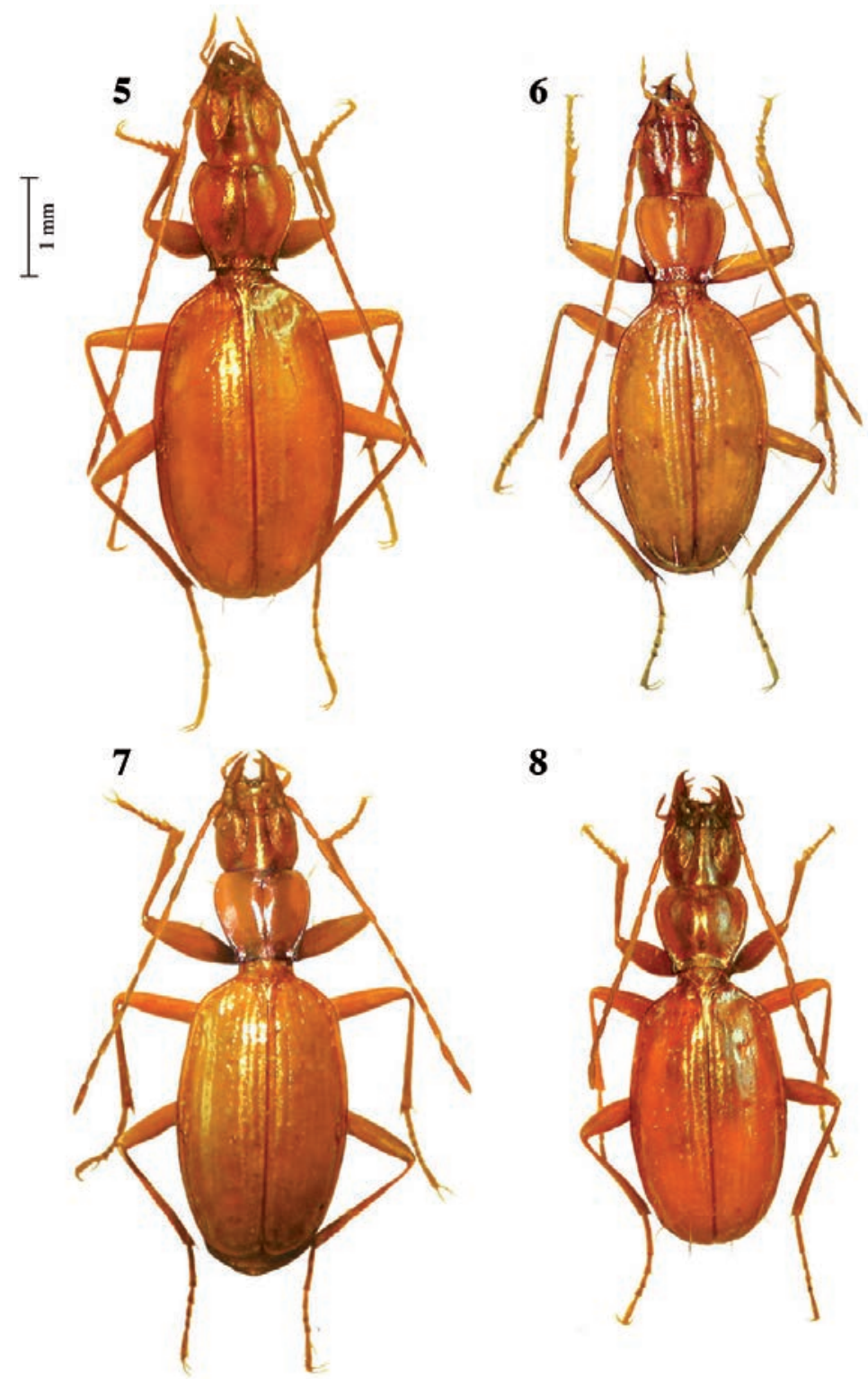

8

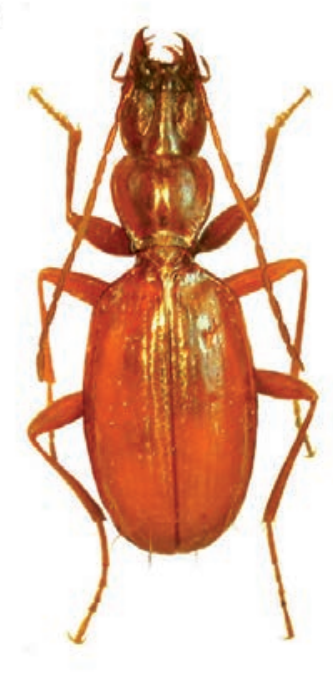

Figg. 5-8. Habitus in visione dorsale di esemplari đô topotipici di: 5 - Boldoriella carminatii; 6 - Boldoriella manzoniana; 7 - Boldoriella silvanae; 8 - Boldoriella concii. 
Tab. 1. Valori biometrici in Boldoriella del gruppo carminatii.

\begin{tabular}{|c|c|c|c|}
\hline & Lunghezza minima totale & Lunghezza massima totale & $\begin{array}{l}\text { Rapporto lunghezza } \\
\text { antenne/corpo }\end{array}$ \\
\hline Boldoriella carminatii & $4.8 \mathrm{~mm}$ & $6.0 \mathrm{~mm}$ & 0.78 \\
\hline Boldoriella carminatii bucciarellii & $5.2 \mathrm{~mm}$ & $6.5 \mathrm{~mm}$ & 0.78 \\
\hline Boldoriella concii & $4.4 \mathrm{~mm}$ & $5.2 \mathrm{~mm}$ & 0.70 \\
\hline Boldoriella concii folinii & $4.5 \mathrm{~mm}$ & $4.7 \mathrm{~mm}$ & 0.68 \\
\hline Boldoriella concii culminicola & $3.8 \mathrm{~mm}$ & $4.5 \mathrm{~mm}$ & 0.65 \\
\hline Boldoriella silanae & $4.6 \mathrm{~mm}$ & $5.6 \mathrm{~mm}$ & 0.75 \\
\hline Boldoriella manzoniana & $4.5 \mathrm{~mm}$ & $5.8 \mathrm{~mm}$ & 0.75 \\
\hline
\end{tabular}

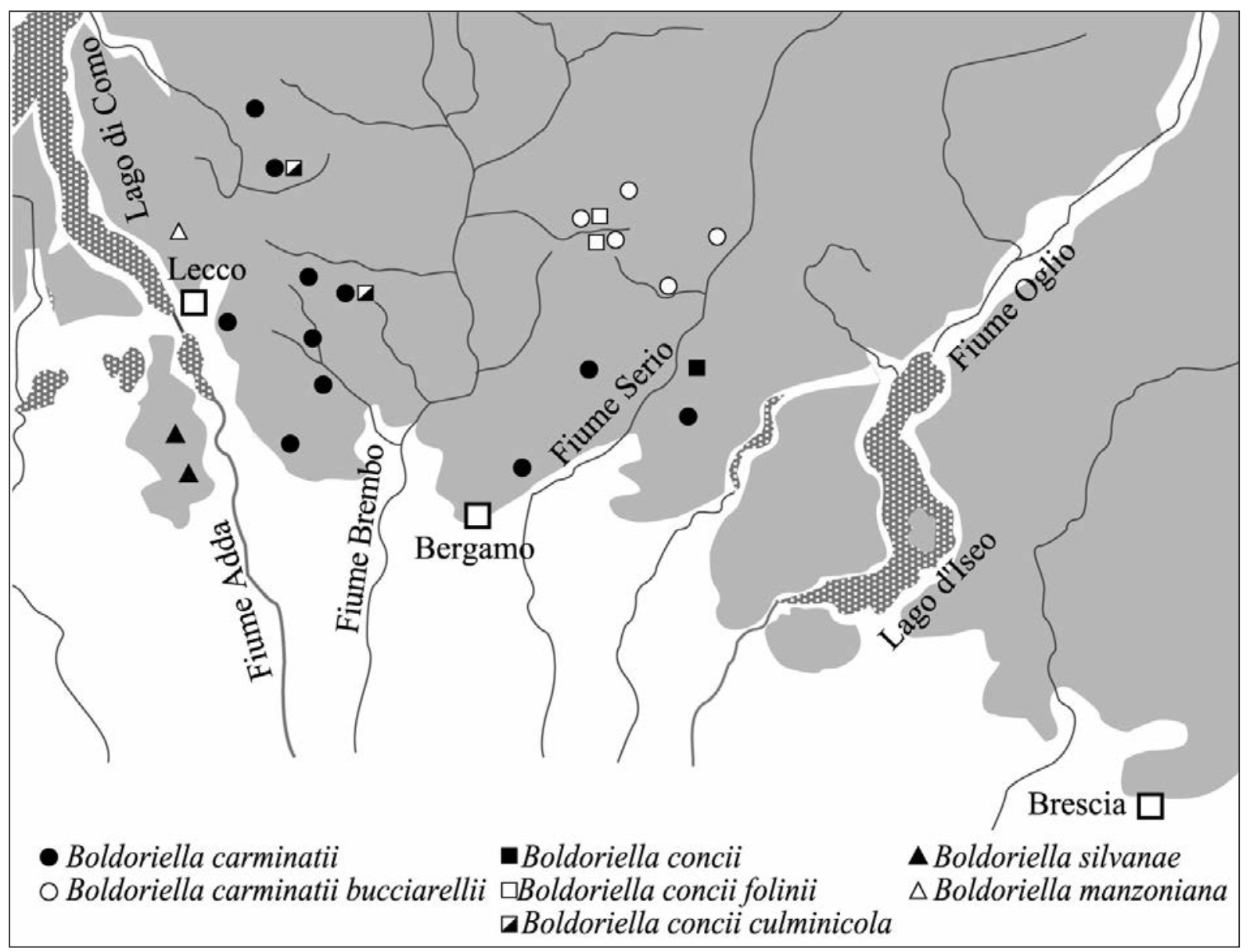

Fig. 9. Cartina delle distribuzioni attualmente note delle specie e sottospecie di Boldoriella del "gruppo carminatii". 
differenzazione nell'alta Val Seriana a nord della barriera dolomitica del Monte Alben, con la sottospecie bucciarellii Monguzzi. Le altre specie del gruppo si aggiungono o si sovrappongono come elementi relitti periferici, confinati in settori ristretti e marginali a volte separati da importanti barriere geologiche come la Valle del Fiume Adda e la Val Sassina.

Delle quattro specie del "gruppo di Boldoriella carminatii", tre risultano simpatriche e sintopiche con altre specie: Boldoriella carminatii con Boldoriella concii, mentre sulle colline dell'alta Brianza, in un ristrettissimo settore montuoso marginale e isolato, separato da una grande e importante valle, quella del Fiume Adda, convivono Boldoriella silvanae con Boldoriella brigantiae (Monguzzi \& Regalin, 2001). Quest'ultima specie mostra anche chiare affinità con $B$. silvanae, ma sembra essere più affine a $B$. pozziae Bari, stenoendemica nel "Triangolo Lariano".

Boldoriella manzoniana è nota di un singolo biotopo costituito dalle miniere del Pian dei Resinelli, scavate in una sella calcarea di scarsa potenza sovrastante le dolomie che costituiscono l'unità strutturale del Monte Coltignone e della Grigna Meridionale, se- parata dalle Orobie occidentali dalla Val Sassina. Questo solco vallivo attualmente costituisce una barriera di scarso rilievo a causa dei profondi rimodellamenti morfologici causati dalle glaciazioni quaternarie (Orombelli et al., 1990); ma prima che questi eventi climatici modificassero il suo assetto originario, la valle costituiva un importante bacino idrografico alimentato dai nevai delle alte cime che lo sovrastano: M.te Legnone, Pizzo dei tre Signori, Grigne e Zuccone Campelli. Il suo corso d'acqua scendeva verso la Pianura Padana, profondamente incassato in un Canyon Messiniano che separava i massicci del complessi Zuccone Campelli-Resegone da un lato, e Grigne-Coltignone dall'altro (Orombelli \& Gnaccolini, 1978).

\section{RINGRAZIAMENTI}

Un sentito ringraziamento ai due anonimi revisori che con suggerimenti e note hanno contribuito a migliorare il manoscritto originale, oltre a Carlo Pesarini del Museo di Storia Naturale di Milano, per la consueta disponibilità e cortesia nel fornire aiuto $\mathrm{e}$ consigli.

\section{BIBLIOGRAFIA}

Boldori L. 1931 - Nuovi appunti sulle larve dei Trechini (nota preliminare). Grotte d'Italia, 5: 1-14, 1 cart.

Casale A. 1973 - Note sui Carabidae (Coleoptera). I. Su alcune specie endogee e troglobie. Bollettino Museo Zoologico Università di Torino, 1973, (1): 1-8.

GHIDINI G.M., 1932 - Osservazioni biologiche sulla larva di Speotrechus carminatii Dod. Bollettino della Società Entomologica Italiana, Genova 64: 42-45.

Dodero A., 1917 - Materiali per lo studio dei Coleotteri italiani con descrizione di nuove specie. Annali Museo civico Storia naturale Giacomo Doria, Genova, 47: 377-386, 5 figg.

Monguzzi R., 1982 - Studi sul genere Boldoriella Jeannel: sistematica, geonemia, ecologia (Col. Carabidae Trechinae). Atti della Società italiana di Scienze naturali, 123 (2-3): 189-236.

Monguzzi R. \& Regalin R., 2001 - Una nuova specie di Boldoriella s. str. dell'alta Brianza (Lombardia) (Coleoptera Carabidae Trechinae). Giornale italiano di Entomologia, 9: 305-311.

Monzini V., 1995 - Nuovi dati geonemici, sistematici e zoogeografici su alcuni Trechini di Lombardia con descrizione di nuove specie (Coleoptera Carabidae). Bollettino della Società Entomologica Italiana, Genova 126 (3): 233-242.

Orombelli G. \& Gnaccolini M., 1978 - Quaderno N. 4 - Composizione petrografica e provenienza del "ceppo" di Paderno d'Adda. Gruppo di studio del quaternario padano. Istituto Geologico, Torino, 7-30.

Orombelli G., Cita M.B., Bini A., Gaetani M., Gelati R., Siletto G.B. \& Tunesi A., 1990 - Guide Geologiche Regionali, Alpi e Prealpi Lombarde (a cura della Società Geologica Italiana - Dipartimento di Scienze della Terra dell’Università di Milano). Be. Ma editrice 54-56, 60-62, 179-195.

Indirizzo dell'autore:

V. Monzini, via M. Polo, 3, 20098 San Giuliano Milanese (MI). E-mail: vittorino.monzini@fastwebnet.it 